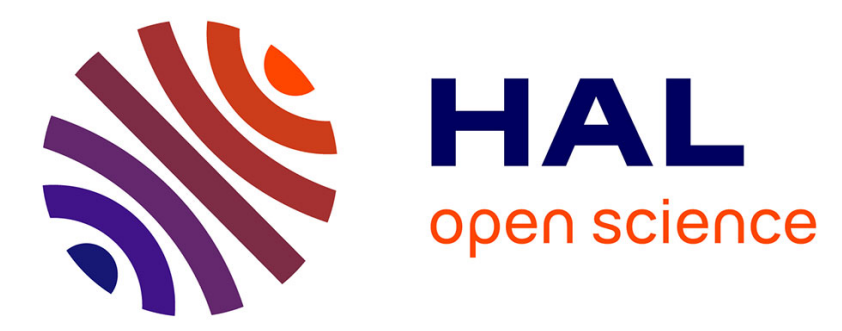

\title{
Automatic quantitative evaluation of image registration techniques with the "epsilon" dissimilarity criterion in the case of retinal images.
}

Yann Gavet, Mathieu Fernandes, Jean-Charles Pinoli

\section{- To cite this version:}

Yann Gavet, Mathieu Fernandes, Jean-Charles Pinoli. Automatic quantitative evaluation of image registration techniques with the "epsilon" dissimilarity criterion in the case of retinal images.. Tenth International Conference on Quality Control by Artificial Vision QVAC 2011, Jun 2011, Saint Etienne, France. pp.8000-23, 10.1117/12.890883 . hal-00614076

\section{HAL Id: hal-00614076 https://hal.science/hal-00614076}

Submitted on 9 Aug 2011

HAL is a multi-disciplinary open access archive for the deposit and dissemination of scientific research documents, whether they are published or not. The documents may come from teaching and research institutions in France or abroad, or from public or private research centers.
L'archive ouverte pluridisciplinaire HAL, est destinée au dépôt et à la diffusion de documents scientifiques de niveau recherche, publiés ou non, émanant des établissements d'enseignement et de recherche français ou étrangers, des laboratoires publics ou privés. 


\title{
Automatic quantitative evaluation of image registration techniques with the $\epsilon$ dissimilarity criterion in the case of retinal images.
}

\author{
Yann Gavet, Mathieu Fernandes and Jean-Charles Pinoli \\ École des Mines de Saint-Étienne, \\ CIS-LPMG/CNRS, \\ 158 Cours Fauriel, 42023 SAINT-ÉTIENNE, FRANCE
}

\begin{abstract}
In human retina observation (with non mydriatic optical microscopes), a registration process is often employed to enlarge the field of view. For the ophthalmologist, this is a way to spare time browsing all the images. A lot of techniques have been proposed to perform this registration process, and indeed, its good evaluation is a question that can be raised.

This article presents the use of the $\epsilon$ dissimilarity criterion to evaluate and compare some classical featurebased image registration techniques. The problem of retina images registration is employed as an example, but it could also be used in other applications. The images are first segmented and these segmentations are registered. The good quality of this registration is evaluated with the $\epsilon$ dissimilarity criterion for 25 pairs of images with a manual selection of control points. This study can be useful in order to choose the type of registration method and to evaluate the results of a new one.
\end{abstract}

Keywords: Image registration, Human retina, Dissimilarity criterion

\section{INTRODUCTION}

An image registration technique consists in representing two or more images into the same coordinate system, such that there is a correspondance between the structures of the images. For more informations, a survey is presented in [1]. When performing an image registration, the question of accuracy is raised. A visual assessment can be performed, but there is not quantification. Some similarity measures can be used [2-4]. This article proposes the use of a novel dissimilarity criterion [5] to evaluate quantitatively the performance of different registration methods. This article is centered on this dissimilarity criterion and is not intended to be a survey on registration techniques. The problem of the quality control on retinal images registration is addressed as an illustration, but the proposed dissimilarity criterion can be employed in a lot of different application areas such as the the control of image segmentation processes [5].

Registration techniques can be divided into two categories: iconic registration and feature-based registration techniques. In the case of retinas, iconic registration is not a good choice because images present different illumination conditions. Moreover, one can notice that the ophthalmologists manually perform this registration process by using a landmark matching based on the vasculature and its bifurcations 6 .

\subsection{Dissimilarity criterion}

The $\epsilon$ dissimilarity criterion is defined as a comparison either between continuous [5] sets or between discrete [5] sets (in this article, the considered sets are discrete, e.g. finite sets of pixels). Practically, these sets can be the results of some image segmentation process. This criterion introduces a tolerance in the measure (thus, it is not mathematically a metric) by the mean of the Minkowski addition.

\footnotetext{
Further author information: (Send correspondence to Y. Gavet)

Y. Gavet: E-mail: gavet@emse.fr, Telephone: [33] 477420170
} 


\subsubsection{Minkowski addition}

The Minkowski addition [7] defines an algebraic operation between sets in $\mathbb{R}^{n}$.

If $M$ and $B$ are two sets of $\mathbb{R}^{n}$, the Minkowski sum of $M$ and $B$, denoted $M \oplus B$, is then defined by:

$$
\begin{aligned}
& M \oplus B=\{m+b \mid m \in M, b \in B\} \\
& M \oplus B=\bigcup_{b \in B}\{m+b \mid m \in M\}
\end{aligned}
$$

where $\oplus$ is the Minkowski addition symbol. In the field of Mathematical Morphology [8], it corresponds to the dilation, and $B$ is called a structuring element (for example a ball).

\subsection{Definition of the dissimilarity criterion $\epsilon$}

The $\epsilon$ dissimilarity criterion with the tolerance $\rho$ applied to segmented (binary) images is defined in the case of discrete (numerical) images by the following equation [5]:

$$
\epsilon_{M}^{\rho}(X)=\frac{\#\{(X \backslash M \oplus \rho N) \cup(M \backslash X \oplus \rho N)\}}{\#\{M \oplus \rho N\}}
$$

with $N$ being the structuring element of radius 1 (the unit ball) and \# denoting the number of pixels in the set (\# is the cardinal operator, counting the number of non null pixels in the sets $X$ or $M$. The reader can see this formula as a tolerant version of the symmetric difference). Practically, $\rho$ is the radius of the ball used to dilate the binary images. The choice of this value is not the purpose of this article, though it remains an important question. The value $\rho=3$ is chosen.

\section{METHOD}

\subsection{Human retina image database}

A database of 25 pairs of retina images has been constructed. For each pair of images, the points of correspondance (called control points) have been manually marked by an expert (a minimum of 15 points of correspondance for each pair). Generally, these points are automatically extracted [9], but this remains a difficult task. The manual selection of the pairs of points thus avoids the difficult problem of their automatic detection.

Additionnaly, the (almost circular) region of interest of the images are extracted (see Fig. 1): this will be used as a mask to constrain the overlapping areas.

\subsection{Image segmentation}

A segmentation process extracts the vessels present in the images. A lot of different methods exists for achieving this task [10-12]. In this article, only one method is employed to get the vessels: the one of Chanwimaluang [12]. Two examples are presented in Fig. 1(c) and 1(d). These segmented vessels will be used later for the quantitative evaluation of some registration techniques.

As we choose to evaluate the registration process by the evaluation of the registration of the segmentation, it is important to notice that some other segmentation evaluation criterions could have also be used 5 . The main drawbacks of these methods is that they are not tolerant to small spatial variations of the segmentated objects.

\subsection{Image registration}

The registration process consists in finding a spatial transformation to be applied to one image to fit the other. Thus, the computation of this transformation is performed on the sets of correspondance points. If $u$ and $v$ are the coordinates of a point in the image to be registered $U$ (as Unregistered), and $x$ and $y$ its coordinates in the second image $B$ (as Base image), the transformation used belongs to one of the following categories presented hereafter (see $[1,13]$ for more details on these methods): 


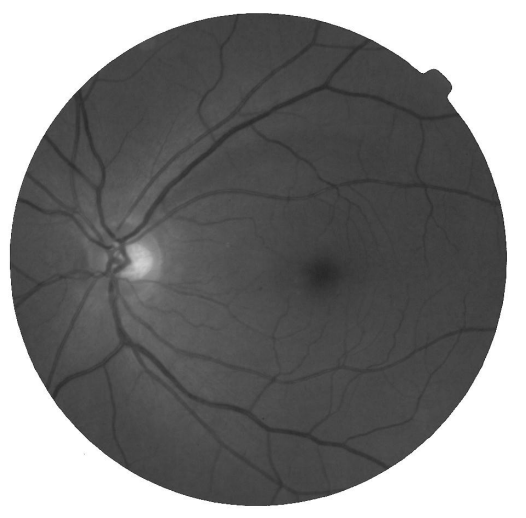

(a)

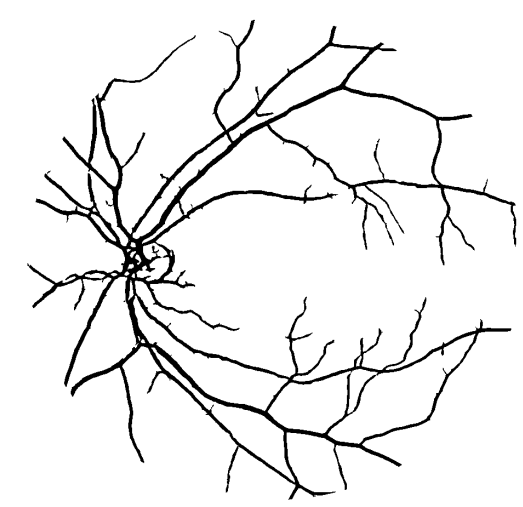

(c)

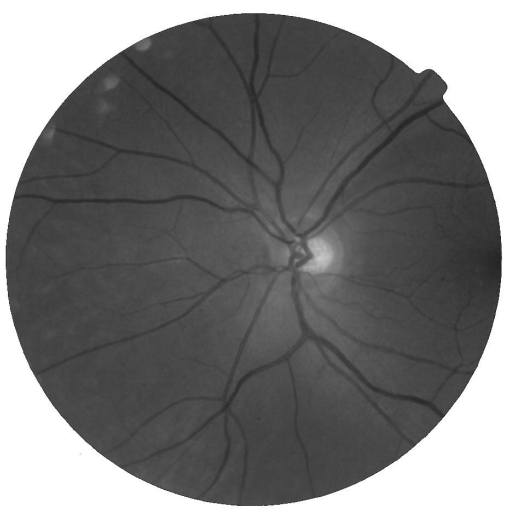

(b)

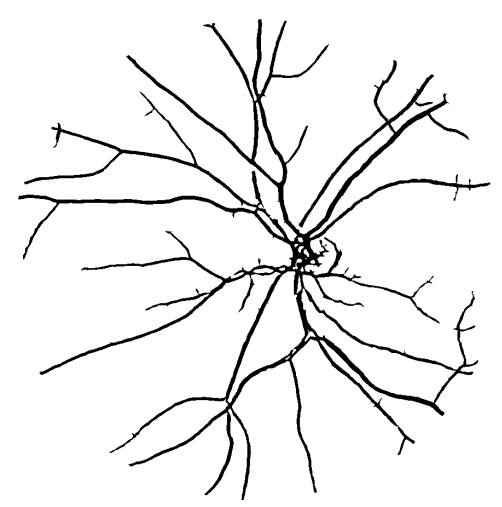

(d)

Figure 1. Example of a pair of retina images. The represented images (a) and (b) are the green component of the RGB images. The binary result of the segmentation (see subsec. 2.2) of these images is respectively represented in (c) and (d) for the images (a) and (b). 
- Similarity: the computed transformation is a similarity (a combination of translation $(h$ and $k$ ), rotation $\theta$ and scaling $s$ ).

$$
\begin{aligned}
& u=x \cdot s \cos \theta-y \cdot s \sin \theta+h \\
& v=x \cdot s \sin \theta+y \cdot s \cos \theta+k
\end{aligned}
$$

- Affine: a first order polynomial relation between the two systems of coordinates.

$$
\begin{aligned}
& u=a x+b y+c \\
& v=d x+e y+f
\end{aligned}
$$

- Projective: this transformation may be used in the case of nonlinearities in the lens or sensor.

$$
\begin{aligned}
u & =\frac{a x+b y+c}{d x+e y+1} \\
v & =\frac{f x+g y+h}{d x+e y+1}
\end{aligned}
$$

- Polynomial: the relation between the coordinates is polynomial. Let $P$ and $Q$ be two polynomials for which the maximum degree in $x$ and $y$ is $n$. Practically, $n$ is 2 or 3 . If $n=1$, the transformation is affine.

$$
\begin{aligned}
& u=P(x, y) \\
& v=Q(x, y)
\end{aligned}
$$

- Piecewise linear [14]: a linear transformation function is used to map a triangle (defined by a Delaunay triangulation of the control points) in the registered image to the corresponding triangle in the base image.

- Local linear: Fernandes et al. [9] proposed a method that computes a transformation for each point in the images from its two nearest control points.

The masks of the images are applied so that only the overlapping regions appear as a result.

\section{QUANTITATIVE EVALUATION AND RESULTS}

The goal of this article, presented in this section, is to propose an answer to the questions: can I control the good quality of a registration process, how good is it, and how can I choose among the different transformations?

\subsection{Mean results over the database}

The registration process is performed on the segmented images of human retinas. Only the overlapping regions are kept (with the use of the masks) and the $\epsilon$ dissimilarity criterion is applied on them. To compare the different methods together, the results are normalized by the results of the affine method, which is considered as a reference. Notice that the lower the criterion value is, the better the registration method is.

Let $B$ be the base (segmented) image, and $U$ the unregistered (segmented) image. The transformation $T$ maps $U$ into the coordinate system of $B$. If $A$ is the affine transformation, the normalized $\epsilon$ criterion, denoted $\tilde{\epsilon}$, is computed as follows:

$$
\tilde{\epsilon}_{B}(M)=\frac{\epsilon_{B}^{3}(T(U))}{\epsilon_{B}^{3}(A(U))}
$$

- $\tilde{\epsilon} \leq 1$ means that the transformation gives a better result than the affine transformation.

- $\tilde{\epsilon} \geq 1$ means that the transformation gives a lower result than the affine transformation.

The results are proposed in Fig. 3 and Tabs. 2 and 1. They show that the second order polynomial method appears better than the others. The local linear method would be a good choice, but present high values in some cases.

These results must be tempered by the fact that they depend on the choice of the control points, this is why the next subsection will try to evaluate their influence. 


\begin{tabular}{|l|r|}
\hline Registration method & Mean $\tilde{\epsilon}$ \\
\hline Affine & 1 \\
Similarity & 0.99 \\
Projective & 0.93 \\
Polynomial of order 2 & 0.68 \\
Polynomial of order 3 & 0.84 \\
Piecewise linear & 0.95 \\
Local linear & 0.86 \\
\hline
\end{tabular}

Table 1. Results over the 25 images of the retina database. The best average value is obtained by the second order polynomial method. The local linear method gives generally good results but sometimes the distortion of the registered images is too strong (see Fig. 3).

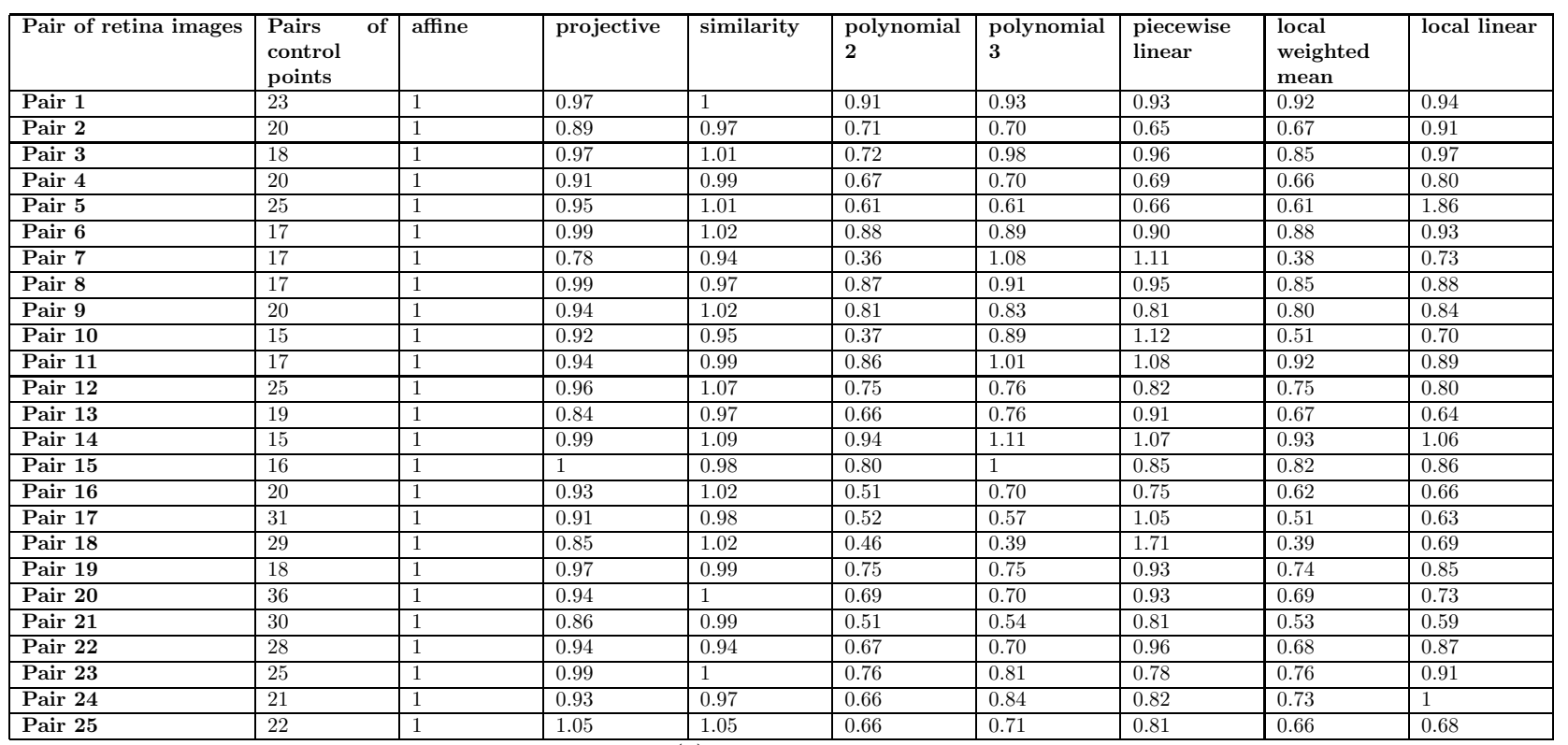

Table 2. This table presents the normalized results $(\tilde{\epsilon})$ for the 25 pairs of retina images and for the different registration methods. The lower values are the best results. The first column presents the number of pairs of control points for each pair of retina images in the database. 


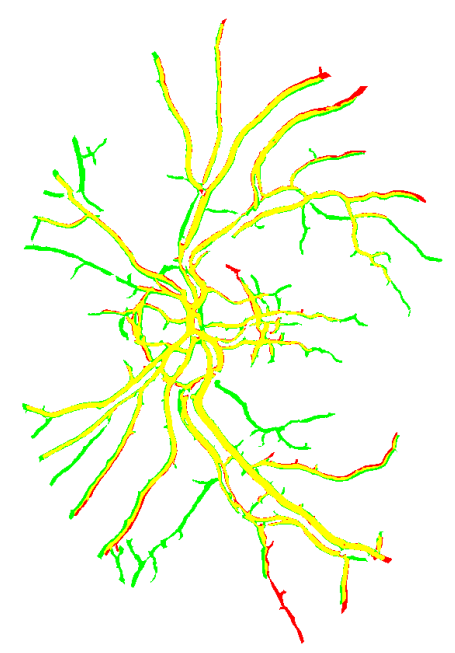

Figure 2. Result of the registration of segmented images of a retina. The masks of the regions of interest have been applied. The base image is represented by the red channel, the registered image is represented by the green channel. The superimposition appears in yellow.

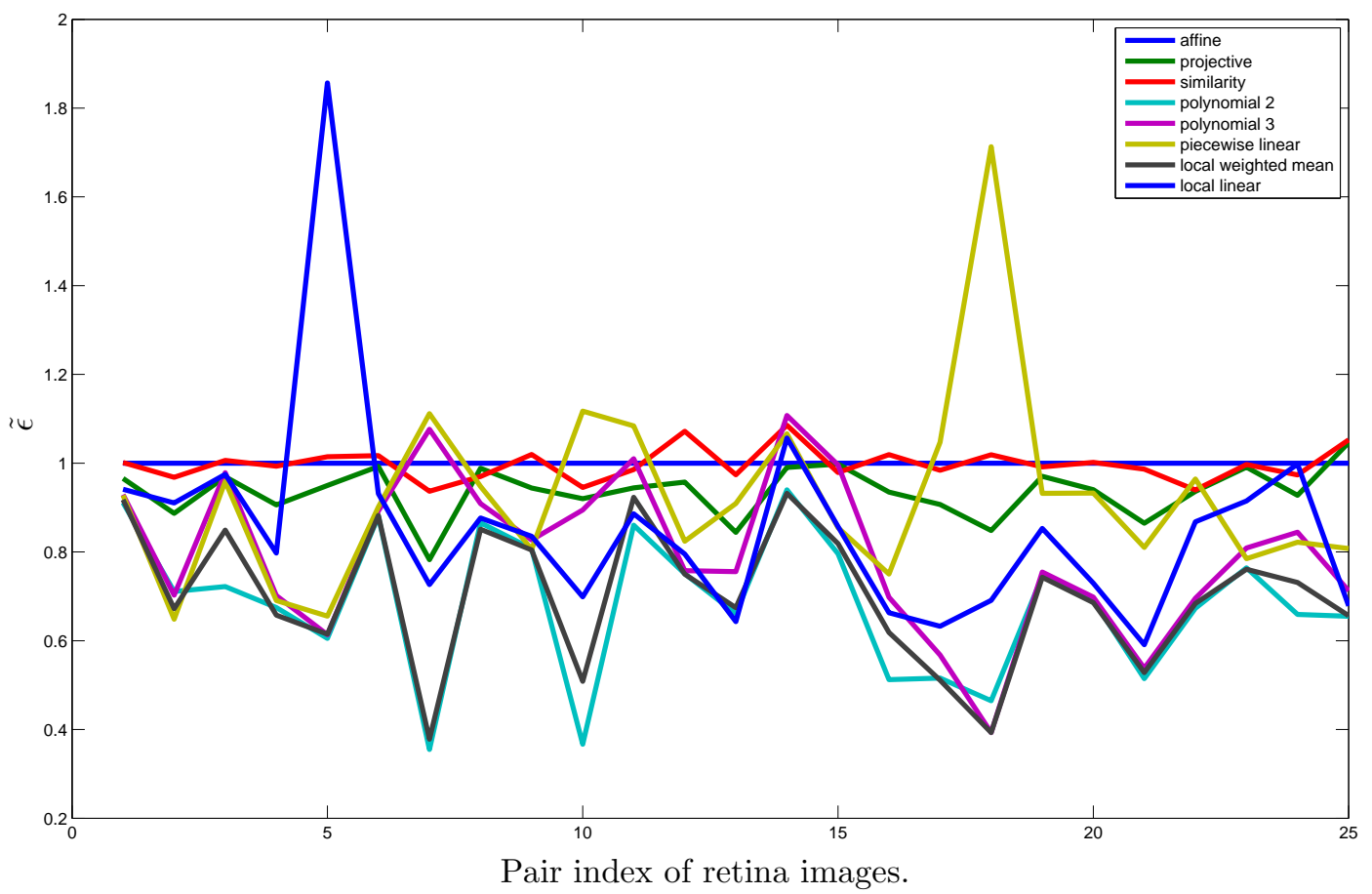

Figure 3. Quantitative comparison between 8 registration techniques using the $\epsilon$ dissimilarity criterion. Evolution of the $\epsilon$ dissimilarity criterion (normalized by the affine registration method) for 25 images of the human retina database. The second order polynomial method gives the best results in average. 


\subsection{Influence of the number of control points}

To evaluate the influence of the number of control points, one pair of corresponding human retina images is taken (Pair number 25). This pair of images contains 22 pairs of control points. The question we want to answer is: what happens if we take only a few control points among those?

The results are presented in Fig. 4. The number of control points is randomly choosen (among 22 points) and the average $\epsilon$ value is represented is abscissa of 100 random choices. $\epsilon_{B}^{3}(T(U)$ ) (measuring the dissimilarity of the transformation of the unregistered image $T(U)$ compared to the base image $B$ ) is represented in ordinates. Notice that each method requires a minimum number of pairs of control points.

It appears (for this pair of images) that the local linear method gives better results even for a low number of control points. This is only when using more than 17 pairs of points that the second order polynomial method gives lowest $\epsilon$ and thus better results.

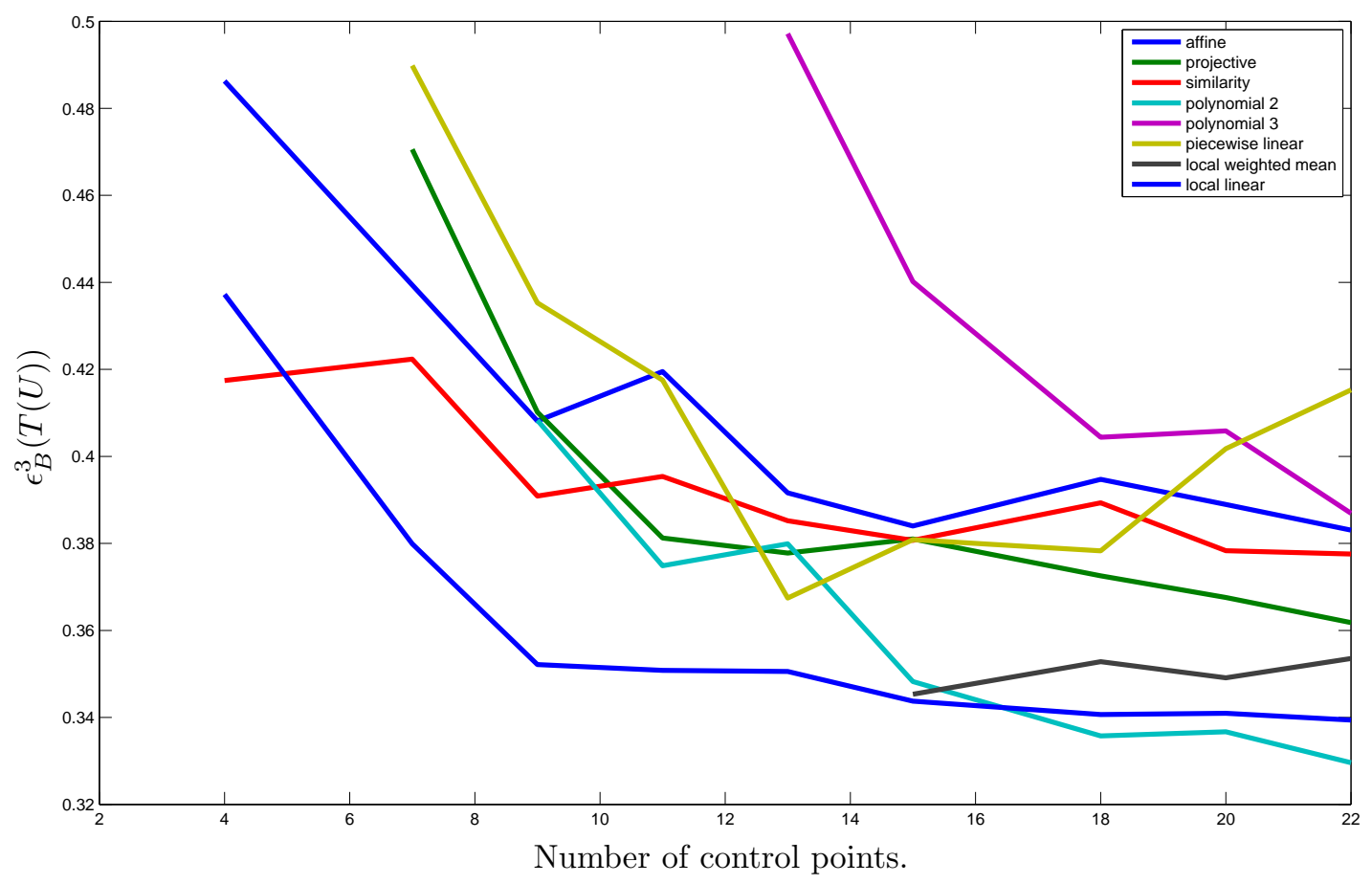

Figure 4. This figure shows the evaluation of the registration in regard to the number of control points. This example is computed for only one pair of retina images. It shows that the so-called local linear method generally performs well even when a small number of points is used.

\section{CONCLUSION AND PERSPECTIVES}

The problem of retinal images registration is adressed as an illustration to the use of the $\epsilon$ dissimilarity criterion. In this article, some classical registration techniques are evaluated and compared. It is shown that the $\epsilon$ criterion is fairly simple to understand and manipulate. The reader can notice that $\epsilon$ could also be employed to evaluate results of segmentation, which was the reason why it was originally introduced [5].

\section{ACKNOWLEDGMENTS}

The authors wish to thank Pr. Gilles Thuret and Pr. Philippe Gain from University Hospital Centre, SaintEtienne, France for supporting this work and for providing pictures of human retinas. 


\section{REFERENCES}

[1] Zitova, B., "Image registration methods: a survey," Image and Vision Computing 21, 977-1000 (October 2003).

[2] Ritter, N., Owens, R., Cooper, J., Eikelboom, R., and Van Saarloos, P., "Registration of stereo and temporal images of the retina," Medical Imaging, IEEE Transactions on 18, 404-418 (May 1999).

[3] Skerl, D., Likar, B., and Pernus, F., "A protocol for evaluation of similarity measures for rigid registration," Medical Imaging, IEEE Transactions on 25(6), 779 -791 (2006).

[4] Lalibert, F., Gagnon, L., and Sheng, Y., "Registration and fusion of retinal images-an evaluation study," Medical Imaging, IEEE Transactions on 22, 661 -673 (May 2003).

[5] Gavet, Y., Fernandes, M., and Pinoli, J.-C., "The $\epsilon$ criterion: a contour-based supervised tolerant image segmentation evaluation.," in [IEEE International Conference on Image Processing], (2011). submitted.

[6] Can, A., Stewart, C. V., Roysam, B., and Tanenbaum, H. L., "A feature-based, robust, hierarchical algorithm for registering pairs of images of the curved human retina," IEEE Transactions on Pattern Analysis and Machine Intelligence 24, 347-364 (2002).

[7] Minkowski, H., "Volumen und Oberfläche," Mathematische Annalen 57, 447-495 (1903).

[8] Soille, P., [Morphological Image Analysis: Principles and Applications], Springer-Verlag New York, Inc., Secaucus, NJ, USA, 2nd ed. (2003).

[9] Fernandes, M., Gavet, Y., and Pinoli, J.-C., "A feature-based dense local registration of pairs of retinal images.," in [Proceedings of the 4th International Conference on Computer VISion Theory and APplications, VISAPP 2009 ISBN=978-989-8111-69-2 VISAPP 2009: 4th International Conference on Computer VISion Theory and APplications], Ranchordas, A. ; Araujo, H., ed., 1, 265-265, INSTICC-Institut for Systems and Technologies of Information Control and Communication, Lisbonne Portugal (2009).

[10] Chaudhuri, S., Chatterjee, S., Katz, N., Nelson, M., and Goldbaum, M., "Detection of blood vessels in retinal images using two-dimensional matched filters," Medical Imaging, IEEE Transactions on 8, 263-269 (sep 1989).

[11] Staal, J., Abramoff, M., Niemeijer, M., Viergever, M., and van Ginneken, B., "Ridge-based vessel segmentation in color images of the retina," Medical Imaging, IEEE Transactions on 23, 501-509 (april 2004).

[12] Chanwimaluang, T., Fan, G., and Fransen, S. R., "Hybrid retinal image registration," IEEE Transactions on Information Technology in Biomedicine 10(1), 129-142 (2006).

[13] IMGFSR, "Transformation functions for image registration," tech. rep., Image Fusion Systems Research.

[14] Goshtasby, A., "Piecewise linear mapping functions for image registration," Pattern Recognition 19(6), 459-466 (1986). 